\title{
Metronomic chemotherapy: an attractive alternative to maximum tolerated dose therapy that can activate anti-tumor immunity and minimize therapeutic resistance
}

\author{
Irina Kareva ${ }^{1,2}$, David J. Waxman ${ }^{3}$, Giannoula L. Klement ${ }^{1}$
}

${ }^{1}$ Newman Lakka Institute, Floating Hospital for Children at Tufts Medical Center, Boston, MA 02111

${ }^{2}$ Mathematical, Computational and Modeling Sciences Center, Arizona State University, Tempe, AZ 85287

${ }^{3}$ Division of Cell and Molecular Biology, Department of Biology, Boston University Boston, MA 02215

\section{*Correspondence should be sent to:}

Giannoula Lakka Klement, MD, FRCPC

Director, Newman Lakka Institute for Personalized Cancer Care

Director, Rare Tumors and Vascular Anomalies Center

Chief, Academic \& Research Affairs, Pediatric Hematology Oncology,

Floating Hospital for Children at Tufts Medical Center.

800 Washington Street, Box 14

Boston, MA 02111, USA

Tel (Clinic): 617 636-5535

Tel (Institute): 617 636-8181

Fax (Clinic): 617 636-7738

Email: glakkaklement@tuftsmedicalcenter.org

(C) 2014. This manuscript version is made available under the Elsevier user license 


\begin{abstract}
The administration of chemotherapy at reduced doses given at regular, frequent time intervals, termed 'metronomic' chemotherapy, presents an alternative to standard maximal tolerated dose (MTD) chemotherapy. The primary target of metronomic chemotherapy was originally identified as endothelial cells supporting the tumor vasculature, and not the tumor cells themselves, consistent with the emerging concept of cancer as a systemic disease involving both tumor cells and their microenvironment. While antiangiogenesis is an important mechanism of action of metronomic chemotherapy, other mechanisms, including activation of anti-tumor immunity and a decrease in acquired therapeutic resistance, have also been identified. Here we present evidence supporting a mechanistic explanation for the improved activity of cancer chemotherapy when administered on a metronomic, rather than an MTD schedule and discuss the implications of these findings for further translation into the clinic.
\end{abstract}

\title{
Introduction
}

Standard clinical protocols for cancer chemotherapy typically employ the maximal drug dose that can be tolerated by the patient. This, in turn, necessitates prolonged time intervals between treatment cycles to allow for normal tissue recovery from the cytotoxic assault, which are ideally designed to maximize tumor cell kill without lethal damage to the patient. This concept of maximum tolerated dose (MTD) chemotherapy derives from the success of treating acute lymphoblastic leukemia (ALL) in children [82]. Childhood ALL is highly responsive to MTD chemotherapy primarily because it represents a rare instance when the leukemic tumor clone can be completely eradicated. This is not always possible in other, more genetically complex leukemias, such as bcrabl and MLL-positive leukemias, where this treatment strategy has not been as successful [74]. Cancers in which MTD chemotherapy has proven to be 
successful rarely have a complex network of activating mutations, and include gestational choriocarcinomas [14, 62], testicular cancer [94], certain germ-cell tumors [62], Hodgkin disease [40] and B-cell non-Hodgkin lymphomas [43, 80]. In contrast, complex cancers, such as sarcomas, breast, prostate, pancreas and lung cancers, are less effectively treated by up front tumor cell eradication using MTD doses, primarily because these cancers engage the host microenvironment extensively [4, 67-69, 78].

In addition to its high toxicity and detrimental effects on the patients' quality of life, MTD chemotherapy is often followed by the development of therapeutic resistance. Particularly in the case of solid tumors, MTD chemotherapy kills off chemotherapy-sensitive cancer cell populations, leaving chemoresistant cells behind to re-colonize the tumor bed, ultimately leading to disease relapse. One strategy to prevent disease relapse has been to develop increasingly intense and thus more toxic drug regimens, including combination chemotherapy regimens, in the hope of achieving more complete a priori eradication of all cancer cells [78], subscribing to the philosophy of "more must be better". However, recent advances in tumor biology point away from focusing on the cytotoxicity of drugs and toward modification of the biology of the tumor using targeted approaches that disengage the tumor microenvironment. This latter approach re-defines the therapeutic goals to aim for prolonged responses rather than the short-term tumor regression responses, which do not necessarily translate into an increase in long-term patient survival.

In contrast to MTD drug regimens, metronomic chemotherapy is characterized by the administration of a cytotoxic agent at a lower, less toxic dose given at regular, more frequent time intervals. A review of clinical trials comparing the effectiveness of metronomic chemotherapy to MTD chemotherapy $[6,13,36,69$, 77] indicates a growing appreciation of the concept. This trend is also evident at www.clinicaltrials.gov, which currently lists over 150 clinical trials of metronomic chemotherapy for various cancers. Of particular interest are results of the CAIRO3 clinical trial [53], which reported highly encouraging results of 
metronomic maintenance treatment in metastatic colorectal cancer patients. Many more clinical trials using metronomic chemotherapy in combination with molecular agents are ongoing and were recently reviewed [13]. The growing popularity of metronomic chemotherapy reflects the common finding that combining standard chemotherapeutic regimens with non-traditional agents, such as anti-angiogenic drugs, proteasome inhibitors and anti-inflammatory agents, while increasing the response rate, may also increase host toxicity beyond the tolerable level. Metronomic chemotherapy has the potential to preserve efficacy while avoiding the increase in toxicity commonly seen when biologic response modifiers are used.

While metronomic chemotherapy may have been extensively reviewed in the literature and its multiple mechanisms of actions have been well debated [5, $6,12,54,55,68,77]$, for most clinicians, metronomic chemotherapy remains a mostly palliative care tool rather than active, upfront therapy. This categorization of metronomic chemotherapy as palliative tool leads to a disregard of its synergism when used in combination with targeted biological agents and infrequent use in active treatment. By reviewing the mechanisms of action of metronomic chemotherapy in this manuscript in detail, we show the benefits of preferentially using low-dose frequent chemotherapy in facilitating the recent shift in clinical oncology from cytotoxic therapies to molecularly based agents. Furthermore, during the recent Fourth Metronomic and Anti-Angiogenic Therapy meeting (June 2014) one of the main topics of discussion was the "lack of a clear understanding of the exact mechanisms of action, optimal dosages and most efficacious metronomic schedules" [13]. In what follows, we present how changing both the timing and the dosage of chemotherapy, metronomic treatment regimens can effectively address other primary drawbacks of MTD, namely, the development of therapeutic resistance and suppression of anti-tumor immune responses. The argument is supported by the recognition that cancer is a disease not solely of cancer cells, but also of the tumor microenvironment, a point increasingly accepted in the scientific literature [38, 39]. 


\section{Cancer as a disease of both tumor cells and their microenvironment}

Genetically complex tumors grow and develop within a dynamic microenvironment derived from the host's tissues. From this perspective, it may be useful to think of tumor cells as parasites that are hosted by tumor-associated endothelial cells (TECs) and stromal cells, including fibroblasts, pericytes, inflammatory cells, and immune cells, all coexisting within the larger "ecosystem" of the human body. Just as in other ecosystems, the survival of the parasitic tumor cells primarily depends not on the state of the entire ecosystem, but on the state of their local host, in this case the tumor microenvironment. Experience with anti-parasitic treatments has shown that effective eradication strategies require high doses of toxic chemicals, however, such doses cannot be reached because of unacceptable damage to the host. Furthermore, parasites often develop drug resistance resulting in decreased efficacy in subsequent rounds of therapy [1]. These same obstacles are encountered in cancer treatment [29, 30], reinforcing the concept that increased toxicity to the target tumor cell does not equate with an overall increase in efficacy. Attacking 'the immediate host', i.e., TECs and other cells within the tumor microenvironment might in fact prove to be a more successful long-term strategy.

\section{Tumor microenvironment and endothelial cells as targets}

To better understand how the tumor microenvironment becomes engaged and modified by malignant disease, one first needs to understand the functionality of the various components of normal tissues. Most tissues reach a level of dynamic equilibrium under normal conditions, and the local microenvironment can be thought of as a dynamic community composed of a multitude of cells of different lineages and functions, including resident cells and responders [10]. Resident tissue cells include supportive cells, such as fibroblasts, pericytes, astrocytes, and health surveillance cells, such as 
histiocytes, macrophages and lymphocytes. The resident cells provide support to the tissue, while the responders ensure tissue maintenance and protection. Responders are recruited to the tissue site in times of acute need, and can be further subdivided into primary (early) responders, such as platelets, lymphocytes and neutrophils, and secondary responders, such as hematopoietic progenitor cells and monocytes. Both primary and secondary responders are summoned when local health surveillance cells are unable to contain the damage, resulting in recruitment of specialized cells of the adaptive immune response to aid the innate immune cells, or when there is a need to repair tissue damage (Figure 1).

In response to wounding, endothelial cells and fibroblasts send out proangiogenic signals that initiate formation of new blood vessels and stimulate tissue repair. This process is largely mediated by coagulation factors and by platelets, which actively sequester growth factors critical for blood vessel formation, including VEGF, bFGF, PDGF, TSP-1, and PF-4 [51]. Contrary to the widely-held view that platelets release angiogenesis regulators en mass, it is now understood that platelet clots retain angiogenesis regulators and create reciprocally interactive concentration gradients of pro - and anti-angiogenic growth factors [44, 51]. The process of angiogenesis is regulated by the creation of sequential concentration gradients in tissues. More specifically, growth factors such as VEGF initiate formation of sprouts [33], while others, such as bFGF, provide signals for endothelial cell proliferation and tube formation [11, 32, 45], followed by vessel stabilization by PDGF and eventually collagen cleavage and vessel pruning, mediated by such stabilizing and angiogenesis inhibiting factors such as TSP-1 and PF-4 [22, 52]. Thus, under normal physiological conditions, angiogenesis is largely limited to wound healing and placental development. In the tumor microenvironment, however, oncogenic stimulators, such as RAS, cmyc, and EGFR, overcome this inhibition of angiogenesis [65, 75, 76], leading to unrestrained release of angiogenesis-promoting signals. This results in continuous formation of new blood vessels that feed the tumor and further contribute to tumor growth, making angiogenesis an important therapeutic target in cancer. 
An important distinction needs to be made between the anti-angiogenic effects of conventional anti-angiogenic drugs, which target individual molecules or signaling pathways, and the anti-angiogenic actions of metronomic chemotherapy, which inhibit the production of growth factors at the source. For instance, bevacizumab, an anti-angiogenic monoclonal antibody, binds to extracellular VEGF, rendering it incapable of activating cell surface VEGF receptors and thus incapable of initiating sprout formation [81]. In contrast, metronomic chemotherapy damages the source of these growth factors, namely, fibroblasts and TECs [28, 48, 79]. Therefore, while metronomic chemotherapy and anti-angiogenic drugs can both induce anti-angiogenesis, the underlying mechanisms are different, with metronomic therapy potentially having more lasting effects due to its targeting the source of vascular growth factors rather than the growth factors themselves.

There are important differences between TECs and normal endothelial cells [42]. TECs, especially those from highly metastatic tumors have more proangiogenic counterpart than TECs from less metastatic tumors or normal endothelial cells. The quiescence of normal endothelial cells is a welldocumented finding and represents the basis of higher intrinsic sensitivity of TECs to cytotoxic drugs. In some cases TECs lose functionality when exposed to cancer chemotherapeutic agents at much lower concentrations than those needed to cause tumor cell damage. Picomolar to nanomolar concentrations of therapeutic agents such as vinblastine [90], taxol [91], carboplatinum [49] and adriamycin [49] show intrinsic toxicities to TECs, whereas much higher doses -typically nanomolar to micromolar levels -- of the same agents are required for tumor cell toxicity.

\section{Lower dosages can decrease the rate of acquired therapeutic resistance}

The use of lower dosages of cytotoxic drugs for attacking TECs and other supporting cells in the tumor microenvironment can have the added benefit of minimizing the induction of acquired therapeutic resistance [47, 49], particularly 
in the setting of combination therapy. Tumors are characterized by high levels of both genotypic and phenotypic intratumoral heterogeneity, and as a consequence, most tumors are likely to contain one or even multiple cancer cell clones that are resistant to even the highest doses of cytotoxic drugs that can be given to a patient. High doses of cytotoxic chemotherapy (i.e., MTD chemotherapy) impose severe selective pressure on a heterogeneous tumor population, thereby killing drug-sensitive tumor cell clones and leading to the selection of the most drug-resistant clones [35, 64].

Consider the schematic dose-response graphs for killing tumor cells and for TECs presented in Figure 2. With some anticancer agents, the minimal dose needed to inflict significant damage to TECs is so low that tumor cells are spared. All tumor cells depend on TECs and on the stromal compartment for proangiogenic signals that recruit the blood vessels needed to access oxygen and nutrients [59, 87], and as consequence, chemotherapeutic drug doses and schedules that selectively target these and other critical cells within the tumor microenvironment can inflict severe damage on both resistant and sensitive tumor cell clones. This weakens the entire tumor cell population without specifically selecting for resistant clones. In some cases the combination of metronomic chemotherapy with anti-angiogenic therapy yields a superior outcome $[9,15,48]$. Low-dose chemotherapy damages TECs, while the direct acting angiogenesis inhibitors interfere with TEC survival signals, preventing regrowth of new blood vessels. TECs may acquire therapeutic resistance [41], but the mechanisms of TEC resistance are different than the mechanisms of tumor cell resistance, indicating a need for multi-targeted approaches.

One of the primary arguments against administering low dose chemotherapy is based on the experience with infectious diseases and antibiotics, where low (inadequate) drug doses leads to selection for antibioticresistant superbugs [21]. However, this concept is not applicable to low-dose metronomic chemotherapy. Whereas antibiotics still act on the bacteria themselves when given at low dosages, low dose chemotherapy primarily affects 
the stromal cells on which the tumor cells rely for support and sustenance. Therefore, the mechanisms that account for the increased resistance of bacteria treated with low dose antibiotics are largely not applicable to cancer chemotherapy.

\section{Metronomic chemotherapy and cancer stem cells (CSCs)}

Another important question concerns the effect of metronomic chemotherapy on cancer stem-like cells (CSCs), i.e. tumor-initiating cells, which are often intrinsically resistant to classic anticancer drugs. In a study of subcutaneous rat C6 gliomas, metronomic chemotherapy in combination with direct anti-angiogenic drug treatment, but not several other treatment regimens, was effective at reducing the CSC population [26]. Further, neither targeted anti-angiogenic therapy nor cytotoxic chemotherapy alone reduced the fraction of CSCs. Furthermore, others found that an MTD regimen followed by metronomic chemotherapy (a chemo-switch schedule), was more effective in blocking metastatic dissemination in an orthotopic pancreatic adenocarcinoma model compared to MTD treatment [92]. An increase in TSP-1 expression and a decrease in the number of CSCs bearing $\mathrm{CD} 133^{+}$and $\mathrm{CD} 133^{+} / \mathrm{CD} 44^{+} / \mathrm{CD} 24^{+}$ markers was also seen, indicating that a cytoreductive MTD regimen followed by metronomic chemotherapy may be a promising strategy for eradicating chemotherapy-resistant CSCs.

\section{More frequent timing can activate the immune system}

Both innate and adaptive immune responses play an important role in keeping cancer progression in check. These responses can be compromised by high dose chemotherapy, which triggers host inflammatory immune response [27] and ablates immune surveillance [97]. However, these deleterious effects on

the patient's immune system can be managed through changes in both dosage 
and timing of chemotherapy, which can lead to stimulation of anti-tumor immunity and suppression of pro-tumor immune responses.

Low-dose chemotherapy can reduce immune suppressive populations of CD4+CD25+ regulatory $T$ cells (Tregs) $[7,31,34]$. However, metronomic administration of chemotherapeutic drugs can also have effects on other subsets of immune cells. For instance, changing the dosage of commonly used chemotherapeutic drugs can affect antigen presenting cells, such as dendritic cells (DCs), which are crucial for activation of adaptive immune responses [85]. In one study, Tanaka and colleagues [84] evaluated and classified chemotherapeutic agents with respect to their effect on DCs and identified a class of drugs that induced DC maturation. Specifically, vinblastine, which is highly suppressive of anti-tumor immunity at high concentrations, promoted maturation of DCs at low concentrations, as indicated by the increased expression of markers such as MHC-II, CD40, CD80 and CD86. In another study [85], vinblastine increased the activity of cytotoxic lymphocytes against mouse B16 melanoma targets, interfering with the otherwise progressive growth of B16 melanoma. Low doses of chemotherapeutic agents also affect myeloid-derived suppressor cells, alleviating suppression of adaptive immune responses and allowing for improved anti-tumor [83]. An extensive review of the effects of specific chemotherapeutic agents on a variety of immune cell subsets has been published [63].

With respect to innate immune responses, Doloff and Waxman [25] demonstrated that dramatic regression of implanted brain tumor xenografts treated with cyclophosphamide on an intermittent, every 6 day metronomic schedule (Q6day cycle) was accompanied by significant recruitment and activation of innate immune cells, specifically, natural killer (NK) cells, dendritic cells and macrophages. Notably, these responses were achieved with little or no anti-angiogenesis. Selective depletion of NK cells using anti-asialo-GM1 antibody resulted in delayed and incomplete tumor regression, which were both reversed following termination of asialo-GM1 antibody treatment. The efficacy of the 
Q6day cycle was hypothesized to reflect the life span of NK cells and perhaps other first-line immune responder cells. More frequent administration of cytotoxic therapy was ineffective in these brain tumors models, where it may interfere with the immune-stimulating effects of the every 6 -day metronomic regimen by inflicting severe damage to the NK cells themselves [17, 95]. Thus, the timing of metronomic chemotherapy appears to be critical: it needs to be sufficiently frequent to activate a strong innate anti-tumor immune response, but it also needs to be sufficiently well-spaced in time to minimize damage to the immune cells recruited to the tumor microenvironment. Further, longer intervals between metronomic drug treatments (cyclophosphamide given every 9 or every 12 days, instead of every 6 days), and drug doses that are too low, can both lead to tumor escape [95], highlighting the importance of regular, repeated drug treatment for an effective innate anti-tumor response. Other studies show that VEGFR2 signaling is essential for metronomic cyclophosphamide to stimulate robust innate immune cell recruitment [24]. See Figure 3B. Moreover, anti-angiogenic drugs that primarily act by a VEGFR2-independent mechanism do not interfere with innate immune cell recruitment, indicating that the interference with immune cell recruitment is not due to the loss of the tumor vasculature [24]. Avoiding damage to the immune surveillance system might be of crucial importance if the growth of a particular tumor type is dependent on its ability to evade anti-tumor immunity. This contention is supported by Young et al. [96], who suggest that in a clinical setting, optimization of exact dosage and timing may need to be adjusted with respect to the patient's immune response and the type of tumor.

Not all tumors respond to the above intermittent, every 6-day metronomic schedule of cyclophosphamide with NK cell recruitment leading to tumor regression, as seen in a KM12 colon carcinoma model [46]. This may reflect the fact that NK cells are rare in human colorectal carcinoma tissues, even in the presence of high levels of chemokines that activate and recruit these cells, with the capacity for NK cell migration into colorectal carcinoma being impaired early during colorectal carcinoma development [37]. The impact of changes in dose 
and schedule of metronomic chemotherapy on the innate immune response is presented in Figure 3.

\section{Clinical implications and future directions}

Metronomic administration of cancer chemotherapeutic drugs holds much promise to address several of the major drawbacks of MTD regimens. These include the emergence of drug resistance, suppression of anti-tumor immunity, toxicity and poor quality of life during therapy. Further, metronomic chemotherapy lowers the financial burden for the patient when compared to targeted therapies [50], while maintaining efficacy. Unfortunately, the time lag between anti-tumor effect and a visible reduction in tumor bulk may in some cases decrease the utility of metronomic chemotherapy for advanced disease. For example, in the care of brain stem glioma, even minimal progression can be lethal to the patient, calling for more drastic intervention with immediate tumor bulk reduction such as surgery or radiation. Similarly, treatment protocols for ALL include a period of high-intensity induction, followed by a milder dose consolidation, followed by 2-3 years of lower-dose, higher-frequency maintenance therapy [88]. This strategy gives $90-95 \%$ survival rates, and any attempts to omit the maintenance therapy yield inferior results $[23,73,93]$. Choi et al. [18] reported encouraging results for a small group of children with tumors of central nervous system treated with upfront high-dose chemotherapy, followed by metronomic maintenance therapy. In that study, 8 of the 10 patients, including six with metastatic disease, continued to have stable clinical and radiographic disease 20 months from the time of diagnosis. Encouraging results were also reported when using metronomic therapy for children with medulloblastoma, with over $65 \%$ survival rates after 24 months [73], warranting further investigation.

In summary, although the long-standing goal of MTD chemotherapy has been immediate tumor shrinkage, an immediate anti-tumor response that leads to recurrence of disease does not improve patient outcomes. Further, as 
illustrated in Figure 4, while the anti-tumor responses to metronomic chemotherapy may be delayed, e.g., due to the time required to ablate tumor blood vessels or activate an anti-tumor immune response, the anti-tumor response is more likely to be sustained $[48,68]$, owing to the decreased selection of resistant tumor cell clones and the suppression of anti-tumor immunity with a decreased likelihood of disease relapse.

More widespread adoption of metronomic chemotherapy as a main up front therapeutic modality will require improved ways to measure therapeutic efficacy, including the identification of biomarkers that can be used to evaluate therapeutic effectiveness. Such biomarkers may already exist, and include cancer antigens CA 15-3 and CA 19-9, prostate-specific antigen, platelet biomarkers [16, 51, 57, 71, 72], serum VEGF and other angiogenic cytokines [86], plasma levels of PDGF-BB [89], thrombospondin-1 expression [3], VEGF SNPs [66] and CD133 gene expression [2], and various immune response genes[98]. There is also an effort to monitor tumor response using ratios of angiogenesis regulators[70]. Further work is needed however to establish the utility of these biomarkers compared to the old paradigm of measuring the effectiveness of MTD chemotherapy by the degree of myelosuppression or tumor shrinkage.

It remains to be determined whether metronomic chemotherapy will ultimately be more effective than MTD-based therapies in the treatment of metastatic disease, although early indicators suggest this may be the case [19, $20,56,58,60,61]$. The introduction and increased use of computational models to assist with identification of patient-specific optimal dosage and timing protocols will also facilitate the implementation of metronomic chemotherapy in the clinic [8]. The body of experimental and clinical evidence, coupled with theoretical considerations, outlined above, point to metronomic chemotherapy as a preferred course of action.

\section{Acknowledgements}


The authors thank the organizers of the Second Annual Workshop on Cancer Systems Biology of Metronomic Chemotherapy 2012 in Boston, Massachusetts, which was devoted to identifying factors that impede the introduction of metronomic chemotherapy to the clinic. This workshop was funded by funded by an ICBP NIH/NCl - U54 CA149233-01 grant to Dr.Lynn Hlatky. The first author of this manuscript (I.K.) was partially supported by the Office of Science (BER), U.S. Department of Energy, under Award Number DE-SC0001434 (to Philip Hahnfeldt, her postdoctoral fellowship mentor). Supported in part by NIH grant CA049248 (to DJW) and NIH/NIGMS R01 GM093050-01A1 (to GLK). The authors thank Clare Lamont for her artistic help with Figure 1. 


\section{Figure legends}

Figure 1. Role of the tumor microenvironment during tumor progression. Therapeutically resistant tumors engage their microenvironment as cancer cells recruit normal tissue cells, such as fibroblasts, pericytes, histiocytes, platelets and hematopoetic progenitor cells. Through engagement and modification of their microenvironment, cancer cells 'simulate' the conditions of a wound, evoking normal physiological responses such as new blood vessel formation without allowing for angiogenesis termination.

Figure 2. Dose-response curves for cancer cells and tumor endothelial cells (TECs). The dosage of therapeutic agents that achieve maximal cell kill of TECs can be orders of magnitude lower than the dosage necessary to inflict significant damage on cancer cells. Therefore, damaging tumor-supporting endothelial cells may inflict equal damage on all tumor cells, effectively preventing selection for resistant cell clones.

Figure 3. Impact of metronomic chemotherapy frequency and dose on antitumor innate immune responses. A) Impact of metronomic frequency on tumor NK cell response. Chemotherapy administration that is too frequent (daily and Q3d in the glioma models studied; [17]) causes ablation of responding NK cells, while chemotherapy administration that is insufficiently frequent (Q9d and Q12d schedules), while initially effective at activating an innate immune response, leads to abrogation of the anti-tumor immune response and tumor escape [95]. In these models, maximal immunostimulatory and therapeutic responses have been observed on a 6-day schedule. B) Metronomic activation of anti-tumor immunity on a 6-day schedule is impaired when VEGFR2 signaling is inhibited $[24,25]$. C) NK cell dose response on a 6-day schedule is dose-dependent: reduction in dose causes insufficient immune stimulation [17], while a dose that is too high increases NK cell mortality [95]. 
Figure 4: Predicted response to changes in tumor size over time after MTD compared to metronomic chemotherapy. MTD therapy induces early onset, short-term tumor regression, which is frequently followed by a relapse.

Metronomic administration of chemotherapy may have a delayed anti-tumor effect; it may initially lead to tumor growth stasis or even an increase in tumor size, followed by a slow but persistent decrease in overall tumor mass, yielding a potentially alarming short-term effect but superior long-term outcome. 


\section{References}

[1] in: "Antibiotic Resistance Questions \& Answers" [Are antibacterial-containing products (soaps, household cleaners, etc.) better for preventing the spread of infection? Does their use add to the problem of resistance?], Atlanta, Georgia, 2011.

[2] G. Allegrini, T. Di Desidero, M.T. Barletta, A. Fioravanti, P. Orlandi, B. Canu, S. Chericoni, F. Loupakis, A. Di Paolo, G. Masi, A. Fontana, S. Lucchesi, G. Arrighi, M. Giusiani, A. Ciarlo, G. Brandi, R. Danesi, R.S. Kerbel, A. Falcone, G. Bocci, Clinical, pharmacokinetic and pharmacodynamic evaluations of metronomic UFT and cyclophosphamide plus celecoxib in patients with advanced refractory gastrointestinal cancers, Angiogenesis, 15 (2012) 275-286. [3] G. Allegrini, A. Falcone, A. Fioravanti, M.T. Barletta, P. Orlandi, F. Loupakis, E. Cerri, G. Masi, A. Di Paolo, R.S. Kerbel, R. Danesi, M. Del Tacca, G. Bocci, A pharmacokinetic and pharmacodynamic study on metronomic irinotecan in metastatic colorectal cancer patients, Br J Cancer, 98 (2008) 1312-1319. [4] N. Andre, S. Abed, D. Orbach, C.A. Alla, L. Padovani, E. Pasquier, J.C. Gentet, A. Verschuur, Pilot study of a pediatric metronomic 4-drug regimen, Oncotarget, 2 (2011) 960-965.

[5] N. Andre, S. Banavali, Y. Snihur, E. Pasquier, Has the time come for metronomics in low-income and middle-income countries?, Lancet Oncol, 14 (2013) e239-248.

[6] N. Andre, M. Carre, E. Pasquier, Metronomics: towards personalized chemotherapy?, Nature reviews. Clinical oncology, 11 (2014) 413-431.

[7] C. Banissi, F. Ghiringhelli, L. Chen, A.F. Carpentier, Treg depletion with a lowdose metronomic temozolomide regimen in a rat glioma model, Cancer Immunol Immunother, 58 (2009) 1627-1634.

[8] D. Barbolosi, J. Ciccolini, C. Meille, X. Elharrar, C. Faivre, B. Lacarelle, N. Andre, F. Barlesi, Metronomics chemotherapy: time for computational decision support, Cancer Chemother Pharmacol, 74 (2014) 647-652.

[9] L. Bello, G. Carrabba, C. Giussani, V. Lucini, F. Cerutti, F. Scaglione, J. Landre, M. Pluderi, G. Tomei, R. Villani, R.S. Carroll, P.M. Black, A. Bikfalvi, Low-dose chemotherapy combined with an antiangiogenic drug reduces human glioma growth in vivo, Cancer Res, 61 (2001) 7501-7506.

[10] M.J. Bissell, W.C. Hines, Why don't we get more cancer? A proposed role of the microenvironment in restraining cancer progression, Nat Med, 17 (2011) 320329.

[11] R. Blanco, H. Gerhardt, VEGF and Notch in tip and stalk cell selection, Cold Spring Harbor perspectives in medicine, 3 (2013) a006569.

[12] G. Bocci, G. Francia, Metronomic Chemotherapy, Springer, 2014.

[13] G. Bouche, N. Andre, S. Banavali, F. Berthold, A. Berruti, G. Bocci, G.

Brandi, U. Cavallaro, S. Cinieri, M. Colleoni, G. Curigliano, T. Di Desidero, A. Eniu, N. Fazio, R. Kerbel, L. Hutchinson, U. Ledzewicz, E. Munzone, E. Pasquier, O. Graciela Scharovsky, Y. Shaked, J. Sterba, M. Villalba, F. Bertolini, Lessons from the Fourth Metronomic and Anti-angiogenic Therapy Meeting, 24-25 June 2014, Milan, Ecancermedicalscience, 8 (2014) 463. 
[14] M. Bower, F.J. Paradinas, R.A. Fisher, S.K. Nicholson, G.J. Rustin, R.H. Begent, K.D. Bagshawe, E.S. Newlands, Placental site trophoblastic tumor: molecular analysis and clinical experience, Clin Cancer Res, 2 (1996) 897-902. [15] T. Browder, C.E. Butterfield, B.M. Kraling, B. Shi, B. Marshall, M.S. O'Reilly, J. Folkman, Antiangiogenic scheduling of chemotherapy improves efficacy against experimental drug-resistant cancer, Cancer Res, 60 (2000) 1878-1886. [16] D. Cervi, T.T. Yip, N. Bhattacharya, V.N. Podust, J. Peterson, A. AbouSlaybi, G.N. Naumov, E. Bender, N. Almog, J.E. Italiano, Jr., J. Folkman, G.L. Klement, Platelet-associated PF-4 as a biomarker of early tumor growth, Blood, 111 (2008) 1201-1207.

[17] C.S. Chen, J.C. Doloff, D.J. Waxman, Intermittent metronomic drug schedule is essential for activating antitumor innate immunity and tumor xenograft regression, Neoplasia, 16 (2014) 84-96.

[18] L.M. Choi, B. Rood, N. Kamani, D. La Fond, R.J. Packer, M.R. Santi, T.J. Macdonald, Feasibility of metronomic maintenance chemotherapy following highdose chemotherapy for malignant central nervous system tumors, Pediatr Blood Cancer, 50 (2008) 970-975.

[19] M. Colleoni, L. Orlando, G. Sanna, A. Rocca, P. Maisonneuve, G. Peruzzotti, R. Ghisini, M.T. Sandri, L. Zorzino, F. Nole, G. Viale, A. Goldhirsch, Metronomic low-dose oral cyclophosphamide and methotrexate plus or minus thalidomide in metastatic breast cancer: antitumor activity and biological effects, Ann Oncol, 17 (2006) 232-238.

[20] M. Colleoni, A. Rocca, M.T. Sandri, L. Zorzino, G. Masci, F. Nole, G.

Peruzzotti, C. Robertson, L. Orlando, S. Cinieri, B.F. de, G. Viale, A. Goldhirsch, Low-dose oral methotrexate and cyclophosphamide in metastatic breast cancer: antitumor activity and correlation with vascular endothelial growth factor levels, Ann Oncol, 13 (2002) 73-80.

[21] J. Davies, D. Davies, Origins and evolution of antibiotic resistance, Microbiology and molecular biology reviews : MMBR, 74 (2010) 417-433. [22] D.W. Dawson, Bouck, N., Thrombospondin as an inhibitor of angiogenesis, in: B.A. Teicher (Ed.) Thrombospodin, Humana Press, Totowa, NJ, 1999, pp. 185-203.

[23] A.W. Dekker, M.B. van't Veer, W. Sizoo, H.L. Haak, J. van der Lelie, G. Ossenkoppele, P.C. Huijgens, H.C. Schouten, P. Sonneveld, R. Willemze, L.F. Verdonck, W.L. van Putten, B. Lowenberg, Intensive postremission chemotherapy without maintenance therapy in adults with acute lymphoblastic leukemia. Dutch Hemato-Oncology Research Group, J Clin Oncol, 15 (1997) 476-482.

[24] J.C. Doloff, C.S. Chen, D.J. Waxman, Anti-tumor innate immunity activated by intermittent metronomic cyclophosphamide treatment of $9 \mathrm{~L}$ brain tumor xenografts is preserved by anti-angiogenic drugs that spare VEGF receptor 2 , Molecular cancer, 13 (2014) 158.

[25] J.C. Doloff, D.J. Waxman, VEGF receptor inhibitors block the ability of metronomically dosed cyclophosphamide to activate innate immunity-induced tumor regression, Cancer Res, 72 (2012) 1103-1115. 
[26] C. Folkins, S. Man, P. Xu, Y. Shaked, D.J. Hicklin, R.S. Kerbel, Anticancer therapies combining antiangiogenic and tumor cell cytotoxic effects reduce the tumor stem-like cell fraction in glioma xenograft tumors, Cancer Res, 67 (2007) 3560-3564.

[27] L. Galluzzi, L. Senovilla, L. Zitvogel, G. Kroemer, The secret ally: immunostimulation by anticancer drugs, Nat Rev Drug Discov, 11 (2012) 215233.

[28] G. Gasparini, Metronomic scheduling: the future of chemotherapy?, Lancet Oncol, 2 (2001) 733-740.

[29] R.A. Gatenby, A change of strategy in the war on cancer, Nature, 459 (2009) 508-509.

[30] R.A. Gatenby, J. Brown, T. Vincent, Lessons from applied ecology: cancer control using an evolutionary double bind, Cancer Res, 69 (2009) 7499-7502. [31] D. Generali, G. Bates, A. Berruti, M.P. Brizzi, L. Campo, S. Bonardi, A. Bersiga, G. Allevi, M. Milani, S. Aguggini, L. Dogliotti, A.H. Banham, A.L. Harris, A. Bottini, S.B. Fox, Immunomodulation of FOXP3+ regulatory T cells by the aromatase inhibitor letrozole in breast cancer patients, Clin Cancer Res, 15 (2009) 1046-1051.

[32] H. Gerhardt, VEGF and endothelial guidance in angiogenic sprouting, Organogenesis, 4 (2008) 241-246.

[33] H. Gerhardt, C. Betsholtz, How do endothelial cells orientate?, EXS, (2005) 3-15.

[34] F. Ghiringhelli, C. Menard, P.E. Puig, S. Ladoire, S. Roux, F. Martin, E. Solary, A. Le Cesne, L. Zitvogel, B. Chauffert, Metronomic cyclophosphamide regimen selectively depletes CD4+CD25+ regulatory $T$ cells and restores $T$ and NK effector functions in end stage cancer patients, Cancer Immunol Immunother, 56 (2007) 641-648.

[35] M. Greaves, C.C. Maley, Clonal evolution in cancer, Nature, 481 (2012) 306313.

[36] C.R. Hafner, Albrecht; Vogt, Thomas, New Indications for Established Drugs: Combined Tumor-Stroma-Targeted Cancer Therapy with PPAR Agonists, COX2 Inhibitors, mTOR Antagonists and Metronomic Chemotherapy Current Cancer Drug Targets, 5 (2005) 393-419.

[37] N. Halama, M. Braun, C. Kahlert, A. Spille, C. Quack, N. Rahbari, M. Koch, J. Weitz, M. Kloor, I. Zoernig, P. Schirmacher, K. Brand, N. Grabe, C.S. Falk, Natural killer cells are scarce in colorectal carcinoma tissue despite high levels of chemokines and cytokines, Clin Cancer Res, 17 (2011) 678-689.

[38] D. Hanahan, R.A. Weinberg, The hallmarks of cancer, Cell, 100 (2000) 5770.

[39] D. Hanahan, R.A. Weinberg, Hallmarks of cancer: the next generation, Cell, 144 (2011) 646-674.

[40] D. Hasenclever, V. Diehl, A prognostic score for advanced Hodgkin's disease. International Prognostic Factors Project on Advanced Hodgkin's Disease, N Engl J Med, 339 (1998) 1506-1514. 
[41] K. Hida, K. Akiyama, N. Ohga, N. Maishi, Y. Hida, Tumour endothelial cells acquire drug resistance in a tumour microenvironment, J Biochem, 153 (2013) 243-249.

[42] K. Hida, N. Ohga, K. Akiyama, N. Maishi, Y. Hida, Heterogeneity of tumor endothelial cells, Cancer science, 104 (2013) 1391-1395.

[43] H.H. Huang, F. Xiao, F.Y. Chen, T. Wang, J.M. Li, J.M. Wang, J.N. Cao, C. Wang, S.H. Zou, Reassessment of the prognostic value of the International Prognostic Index and the revised International Prognostic Index in patients with diffuse large B-cell lymphoma: A multicentre study, Experimental and therapeutic medicine, 4 (2012) 475-480.

[44] J.E. Italiano, Jr., J.L. Richardson, S. Patel-Hett, E. Battinelli, A. Zaslavsky, S. Short, S. Ryeom, J. Folkman, G.L. Klement, Angiogenesis is regulated by a novel mechanism: pro- and antiangiogenic proteins are organized into separate platelet alpha granules and differentially released, Blood, 111 (2008) 1227-1233. [45] L. Jakobsson, K. Bentley, H. Gerhardt, VEGFRs and Notch: a dynamic collaboration in vascular patterning, Biochemical Society transactions, 37 (2009) 1233-1236.

[46] L. Jia, D.J. Waxman, Thrombospondin-1 and pigment epithelium-derived factor enhance responsiveness of KM12 colon tumor to metronomic cyclophosphamide but have disparate effects on tumor metastasis, Cancer Lett, 330 (2013) 241-249.

[47] R.S. Kerbel, Inhibition of Tumor Angiogenesis as a Strategy to Circumvent Acquired Resistance to Anti-Cancer Therapeutic Agents, BioEssays, 13 (1991) 31-36.

[48] G. Klement, S. Baruchel, J. Rak, S. Man, K. Clark, D.J. Hicklin, P. Bohlen, R.S. Kerbel, Continuous low-dose therapy with vinblastine and VEGF receptor-2 antibody induces sustained tumor regression without overt toxicity, J Clin Invest, 105 (2000) R15-24.

[49] G. Klement, P. Huang, B. Mayer, S.K. Green, S. Man, P. Bohlen, D. Hicklin, R.S. Kerbel, Differences in therapeutic indexes of combination metronomic chemotherapy and an anti-VEGFR-2 antibody in multidrug-resistant human breast cancer xenografts, Clin Cancer Res, 8 (2002) 221-232.

[50] G.L. Klement, B.A. Kamen, Nontoxic, fiscally responsible, future of oncology: could it be beginning in the Third World?, Journal of pediatric hematology/oncology, 33 (2011) 1-3.

[51] G.L. Klement, T.T. Yip, F. Cassiola, L. Kikuchi, D. Cervi, V. Podust, J.E. Italiano, E. Wheatley, A. Abou-Slaybi, E. Bender, N. Almog, M.W. Kieran, J. Folkman, Platelets actively sequester angiogenesis regulators, Blood, 113 (2009) 2835-2842.

[52] G.S.E.V.D. Klement, The role of platelets in angiogenesis, in: A. Michelson (Ed.) Platelets, Elsevier/Academic Press, San Diego, CA, 2012.

[53] M. Koopman, L. Simkens, A. May, L. Mol, H.V. Tinteren, C.J.A. Punt, Final results and subgroup analyses of the phase $3 \mathrm{CAIRO} 3$ study: Maintenance treatment with capecitabine and bevacizumab versus observation after induction treatment with chemotherapy and bevacizumab in metastatic colorectal cancer (mCRC). in: 2014 Gastrointestinal cancers Symposium, 2014. 
[54] K. Lien, S. Georgsdottir, L. Sivanathan, K. Chan, U. Emmenegger, Low-dose metronomic chemotherapy: a systematic literature analysis, Eur J Cancer, 49 (2013) 3387-3395.

[55] R. Maiti, Metronomic chemotherapy, Journal of pharmacology \& pharmacotherapeutics, 5 (2014) 186-192.

[56] L. Manso, N. Valdiviezo, J. Sepulveda, E. Ciruelos, C. Mendiola, I. Ghanem, E. Vega, R. Manneh, M. Dorta, H. Cortes-Funes, Safety and efficacy of metronomic non-pegylated liposomal encapsulated doxorubicin in heavily pretreated advanced breast cancer patients, Clin Transl Oncol, 15 (2013) 467471.

[57] E.L. Mayer, S.J. Isakoff, G. Klement, S.R. Downing, W.Y. Chen, K. Hannagan, R. Gelman, E.P. Winer, H.J. Burstein, Combination antiangiogenic therapy in advanced breast cancer: a phase 1 trial of vandetanib, a VEGFR inhibitor, and metronomic chemotherapy, with correlative platelet proteomics, Breast Cancer Res Treat, 136 (2012) 169-178.

[58] E. Montagna, G. Cancello, V. Bagnardi, D. Pastrello, S. Dellapasqua, G. Perri, G. Viale, P. Veronesi, A. Luini, M. Intra, A. Calleri, C. Rampinelli, A. Goldhirsch, F. Bertolini, M. Colleoni, Metronomic chemotherapy combined with bevacizumab and erlotinib in patients with metastatic HER2-negative breast cancer: clinical and biological activity, Clin Breast Cancer, 12 (2012) 207-214. [59] M.M. Mueller, N.E. Fusenig, Friends or foes - bipolar effects of the tumour stroma in cancer, Nat Rev Cancer, 4 (2004) 839-849.

[60] R. Munoz, S. Man, Y. Shaked, C.R. Lee, J. Wong, G. Francia, R.S. Kerbel, Highly efficacious nontoxic preclinical treatment for advanced metastatic breast cancer using combination oral UFT-cyclophosphamide metronomic chemotherapy, Cancer Res, 66 (2006) 3386-3391.

[61] R. Munoz, Y. Shaked, F. Bertolini, U. Emmenegger, S. Man, R.S. Kerbel, Anti-angiogenic treatment of breast cancer using metronomic low-dose chemotherapy, Breast, 14 (2005) 466-479.

[62] N. Murugaesu, P. Schmid, G. Dancey, R. Agarwal, L. Holden, I. McNeish, P.M. Savage, E.S. Newlands, G.J. Rustin, M.J. Seckl, Malignant ovarian germ cell tumors: identification of novel prognostic markers and long-term outcome after multimodality treatment, J Clin Oncol, 24 (2006) 4862-4866.

[63] M.S. Nars, R. Kaneno, Immunomodulatory effects of low dose chemotherapy and perspectives of its combination with immunotherapy, Int J Cancer, 132 (2013) 2471-2478.

[64] P.C. Nowell, The clonal evolution of tumor cell populations, Science, 194 (1976) 23-28.

[65] F. Okada, J.W. Rak, B.S. Croix, B. Lieubeau, M. Kaya, L. Roncari, S.

Shirasawa, T. Sasazuki, R.S. Kerbel, Impact of oncogenes in tumor angiogenesis: mutant K-ras up-regulation of vascular endothelial growth factor/vascular permeability factor is necessary, but not sufficient for tumorigenicity of human colorectal carcinoma cells, Proc Natl Acad Sci U S A, 95 (1998) 3609-3614.

[66] P. Orlandi, A. Fontana, A. Fioravanti, T. Di Desidero, L. Galli, L. Derosa, B. Canu, R. Marconcini, E. Biasco, A. Solini, G. Francia, R. Danesi, A. Falcone, G. 
Bocci, VEGF-A polymorphisms predict progression-free survival among advanced castration-resistant prostate cancer patients treated with metronomic cyclophosphamide, Br J Cancer, 109 (2013) 957-964.

[67] E. Pasquier, N. Andre, New therapeutic advances and perspectives in tumour angiogenesis, Curr Cancer Drug Targets, 10 (2010) 877-878.

[68] E. Pasquier, M. Kavallaris, N. Andre, Metronomic chemotherapy: new rationale for new directions, Nature reviews. Clinical oncology, 7 (2010) 455-465. [69] E. Pasquier, M.W. Kieran, J. Sterba, Y. Shaked, S. Baruchel, O. Oberlin, M.S. Kivivuori, A. Peyrl, M. Diawarra, M. Casanova, S. Zacharoulis, G. Vassal, F. Berthold, A. Verschuur, N. Andre, Moving forward with metronomic chemotherapy: meeting report of the 2nd International Workshop on Metronomic and Anti-Angiogenic Chemotherapy in Paediatric Oncology, Transl Oncol, 4 (2011) 203-211.

[70] H.A. Perroud, M.J. Rico, C.M. Alasino, S.M. Pezzotto, V.R. Rozados, O.G. Scharovsky, Association between baseline VEGF/sVEGFR-2 and VEGF/TSP-1 ratios and response to metronomic chemotherapy using cyclophosphamide and celecoxib in patients with advanced breast cancer, Indian journal of cancer, 50 (2013) 115-121.

[71] J.E. Peterson, D. Zurakowski, J.E. Italiano, Jr., L.V. Michel, S. Connors, M. Oenick, R.J. D'Amato, G.L. Klement, J. Folkman, VEGF, PF4 and PDGF are elevated in platelets of colorectal cancer patients, Angiogenesis, 15 (2012) 265273.

[72] J.E. Peterson, D. Zurakowski, J.E. Italiano, Jr., L.V. Michel, L. Fox, G.L. Klement, J. Folkman, Normal ranges of angiogenesis regulatory proteins in human platelets, American journal of hematology, 85 (2010) 487-493.

[73] A. Peyrl, M. Chocholous, M.W. Kieran, A.A. Azizi, C. Prucker, T. Czech, K. Dieckmann, M.T. Schmook, C. Haberler, U. Leiss, I. Slavc, Antiangiogenic metronomic therapy for children with recurrent embryonal brain tumors, Pediatr Blood Cancer, 59 (2012) 511-517.

[74] P.A. Pizzo, Poplack,D.G., Principles and practice of pediatric oncology, 6 ed., Lippincott Williams \& Wilkins, Philadelphia, 2010.

[75] J. Rak, J. Filmus, G. Finkenzeller, S. Grugel, D. Marme, R.S. Kerbel, Oncogenes as inducers of tumor angiogenesis, Cancer Metastasis Rev, 14 (1995) 263-277.

[76] J. Rak, Y. Mitsuhashi, C. Sheehan, A. Tamir, A. Viloria-Petit, J. Filmus, S.J. Mansour, N.G. Ahn, R.S. Kerbel, Oncogenes and tumor angiogenesis:

differential modes of vascular endothelial growth factor up-regulation in rastransformed epithelial cells and fibroblasts, Cancer Res, 60 (2000) 490-498. [77] A. Romiti, M.C. Cox, I. Sarcina, R. Di Rocco, C. D'Antonio, V. Barucca, P. Marchetti, Metronomic chemotherapy for cancer treatment: a decade of clinical studies, Cancer Chemother Pharmacol, 72 (2013) 13-33.

[78] P. Savage, J. Stebbing, M. Bower, T. Crook, Why does cytotoxic chemotherapy cure only some cancers?, Nat Clin Pract Oncol, 6 (2009) 43-52. [79] O.G. Scharovsky, L.E. Mainetti, V.R. Rozados, Metronomic chemotherapy: changing the paradigm that more is better, Current oncology, 16 (2009) 7-15. 
[80] L.H. Sehn, B. Berry, M. Chhanabhai, C. Fitzgerald, K. Gill, P. Hoskins, R. Klasa, K.J. Savage, T. Shenkier, J. Sutherland, R.D. Gascoyne, J.M. Connors, The revised International Prognostic Index (R-IPI) is a better predictor of outcome than the standard IPI for patients with diffuse large B-cell lymphoma treated with R-CHOP, Blood, 109 (2007) 1857-1861.

[81] T. Shih, C. Lindley, Bevacizumab: an angiogenesis inhibitor for the treatment of solid malignancies, Clin Ther, 28 (2006) 1779-1802.

[82] H.E. Skipper, F.M. Schabel, Jr., L.B. Mellett, J.A. Montgomery, L.J. Wilkoff, H.H. Lloyd, R.W. Brockman, Implications of biochemical, cytokinetic, pharmacologic, and toxicologic relationships in the design of optimal therapeutic schedules, Cancer Chemother Rep, 54 (1970) 431-450.

[83] E. Suzuki, V. Kapoor, A.S. Jassar, L.R. Kaiser, S.M. Albelda, Gemcitabine selectively eliminates splenic Gr-1+/CD11b+ myeloid suppressor cells in tumorbearing animals and enhances antitumor immune activity, Clin Cancer Res, 11 (2005) 6713-6721.

[84] H. Tanaka, H. Matsushima, N. Mizumoto, A. Takashima, Classification of chemotherapeutic agents based on their differential in vitro effects on dendritic cells, Cancer Res, 69 (2009) 6978-6986.

[85] H. Tanaka, H. Matsushima, A. Nishibu, B.E. Clausen, A. Takashima, Dual therapeutic efficacy of vinblastine as a unique chemotherapeutic agent capable of inducing dendritic cell maturation, Cancer Res, 69 (2009) 6987-6994.

[86] J.H. Tang, J.H. Zhao, J.W. Lu, F. Yan, J.W. Qin, B. Xu, Circulating levels of angiogenic cytokines in advanced breast cancer patients with system chemotherapy and their potential value in monitoring disease course, $\mathrm{J}$ Cancer Res Clin Oncol, 137 (2011) 55-63.

[87] T.D. Tlsty, L.M. Coussens, Tumor stroma and regulation of cancer development, Annual review of pathology, 1 (2006) 119-150.

[88] Y. Toyoda, A. Manabe, M. Tsuchida, R. Hanada, K. Ikuta, Y. Okimoto, A. Ohara, Y. Ohkawa, T. Mori, K. Ishimoto, T. Sato, T. Kaneko, M. Maeda, K. Koike, T. Shitara, Y. Hoshi, R. Hosoya, Y. Tsunematsu, F. Bessho, S. Nakazawa, T. Saito, Six months of maintenance chemotherapy after intensified treatment for acute lymphoblastic leukemia of childhood, J Clin Oncol, 18 (2000) 1508-1516. [89] G. Treiber, T. Wex, P. Malfertheiner, Impact of different anticancer regimens on biomarkers of angiogenesis in patients with advanced hepatocellular cancer, $\mathrm{J}$ Cancer Res Clin Oncol, 135 (2009) 271-281.

[90] A. Vacca, M. lurlaro, D. Ribatti, M. Minischetti, B. Nico, R. Ria, A. Pellegrino, $\mathrm{F}$. Dammacco, Antiangiogenesis is produced by nontoxic doses of vinblastine, Blood, 94 (1999) 4143-4155.

[91] A. Vacca, D. Ribatti, M. Iurlaro, F. Merchionne, B. Nico, R. Ria, F. Dammacco, Docetaxel versus paclitaxel for antiangiogenesis, J Hematother Stem Cell Res, 11 (2002) 103-118.

[92] M. Vives, M.M. Ginesta, K. Gracova, M. Graupera, O. Casanovas, G. Capella, T. Serrano, B. Laquente, F. Vinals, Metronomic chemotherapy following the maximum tolerated dose is an effective anti-tumour therapy affecting angiogenesis, tumour dissemination and cancer stem cells, Int J Cancer, 133 (2013) 2464-2472. 
[93] M. Wernli, A. Tichelli, V. von Fliedner, G. Brun del Re, B. Chapuis, M.F. Fey, M. Fopp, J. Gmur, J.P. Grob, E. Jacky, et al., Intensive induction/consolidation therapy without maintenance in adult acute lymphoblastic leukaemia: a pilot assessment. Working Party on Leukaemia of the Swiss Group for Epidemiologic and Clinical Cancer Research (SAKK), Br J Haematol, 87 (1994) 39-43. [94] S.D. Williams, R. Birch, L.H. Einhorn, L. Irwin, F.A. Greco, P.J. Loehrer, Treatment of disseminated germ-cell tumors with cisplatin, bleomycin, and either vinblastine or etoposide, N Engl J Med, 316 (1987) 1435-1440.

[95] J. Wu, D.J. Waxman, Metronomic cyclophosphamide schedule-dependence of innate immune cell recruitment and tumor regression in an implanted glioma model, Cancer Lett, 353 (2014) 272-280.

[96] S.D. Young, M. Whissell, J.C. Noble, P.O. Cano, P.G. Lopez, C.J. Germond, Phase II clinical trial results involving treatment with low-dose daily oral cyclophosphamide, weekly vinblastine, and rofecoxib in patients with advanced solid tumors, Clin Cancer Res, 12 (2006) 3092-3098.

[97] L. Zitvogel, L. Apetoh, F. Ghiringhelli, G. Kroemer, Immunological aspects of cancer chemotherapy, Nat Rev Immunol, 8 (2008) 59-73.

[98] A. Zloza, D.W. Kim, S. Kim-Schulze, M.C. Jagoda, V. Monsurro, F.M. Marincola, H.L. Kaufman, Immunoglobulin-like transcript 2 (ILT2) is a biomarker of therapeutic response to oncolytic immunotherapy with vaccinia viruses, Journal for immunotherapy of cancer, 2 (2014) 1. 


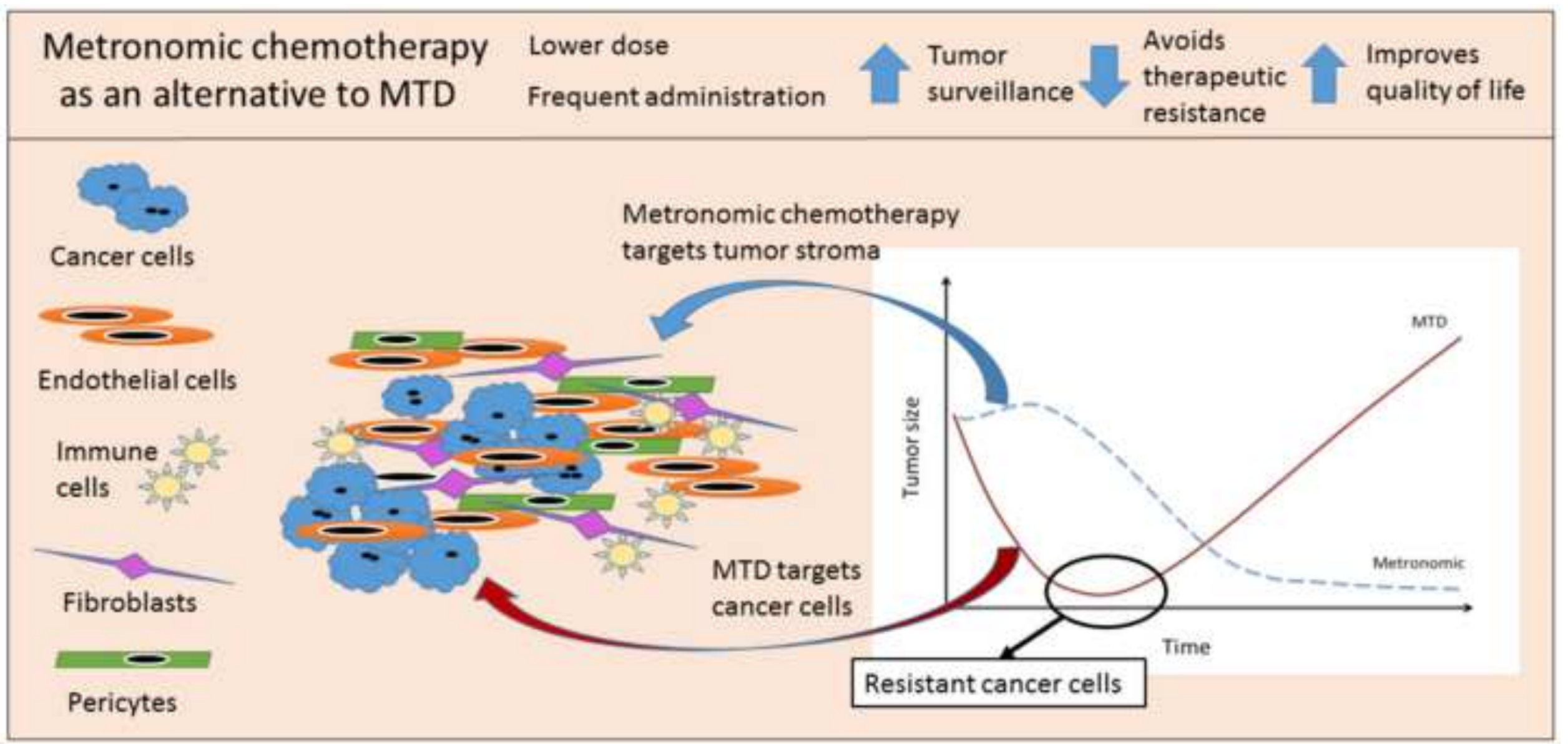


$\therefore$ Cytokines
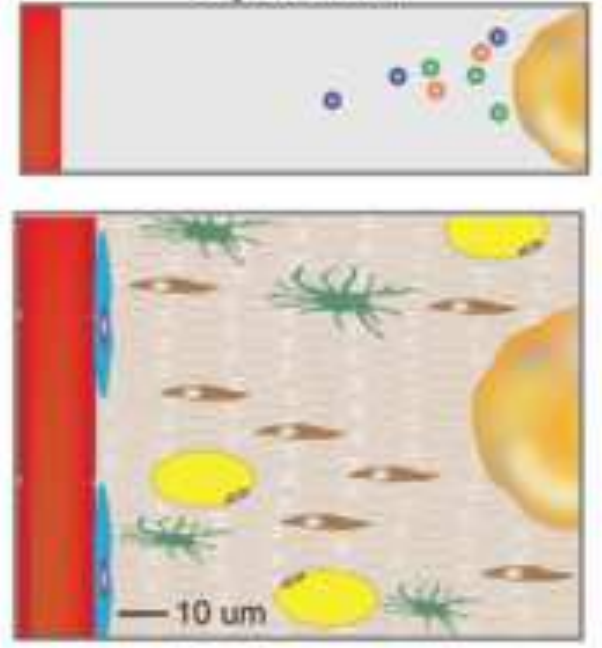

Resident cells

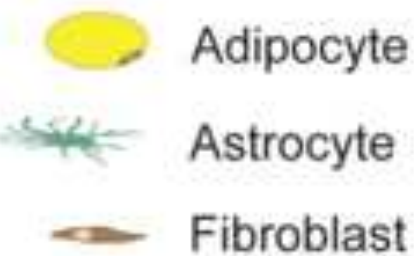

- Pericyte
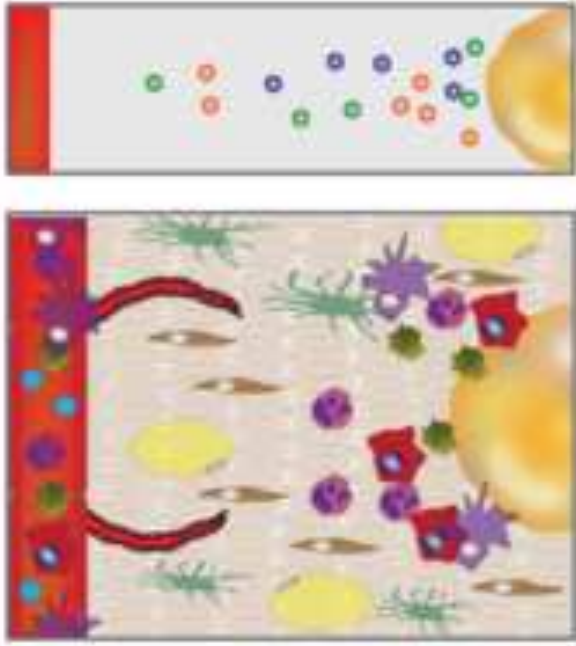

Early responders

$-10 \mathrm{um}$

Macrophage

CHistiocyte

- Lymphocyte

- Neutrophil

- Platelet
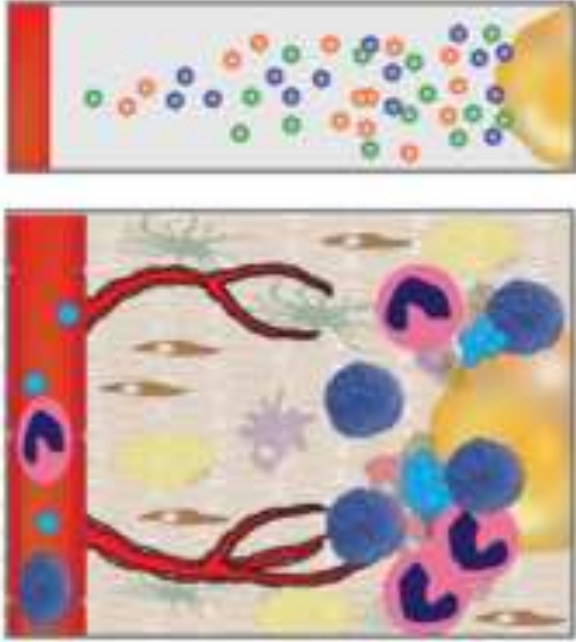

Late responders

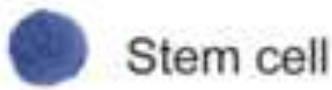

D. Monocyte

\$ Platelet clot 


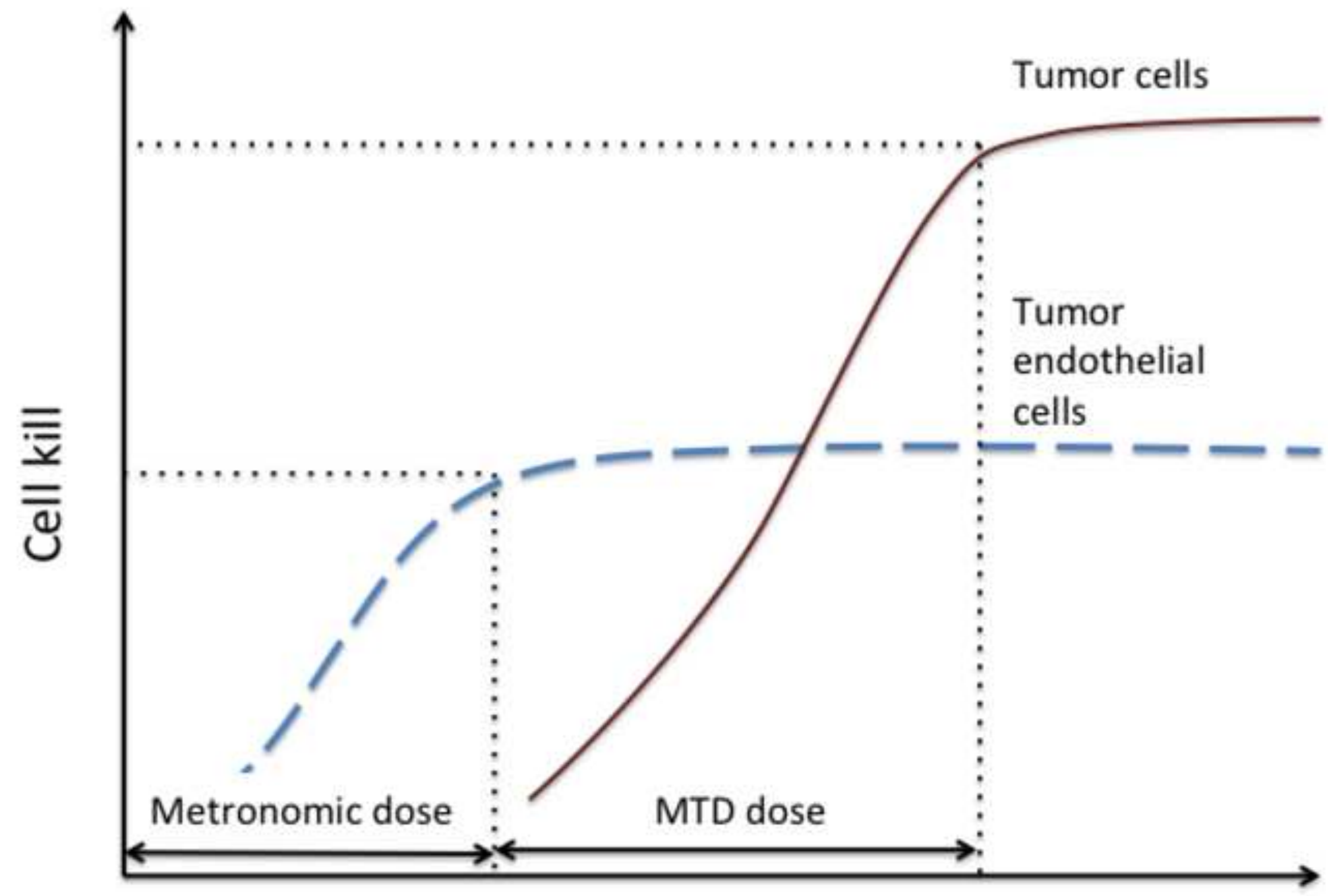

Dose 


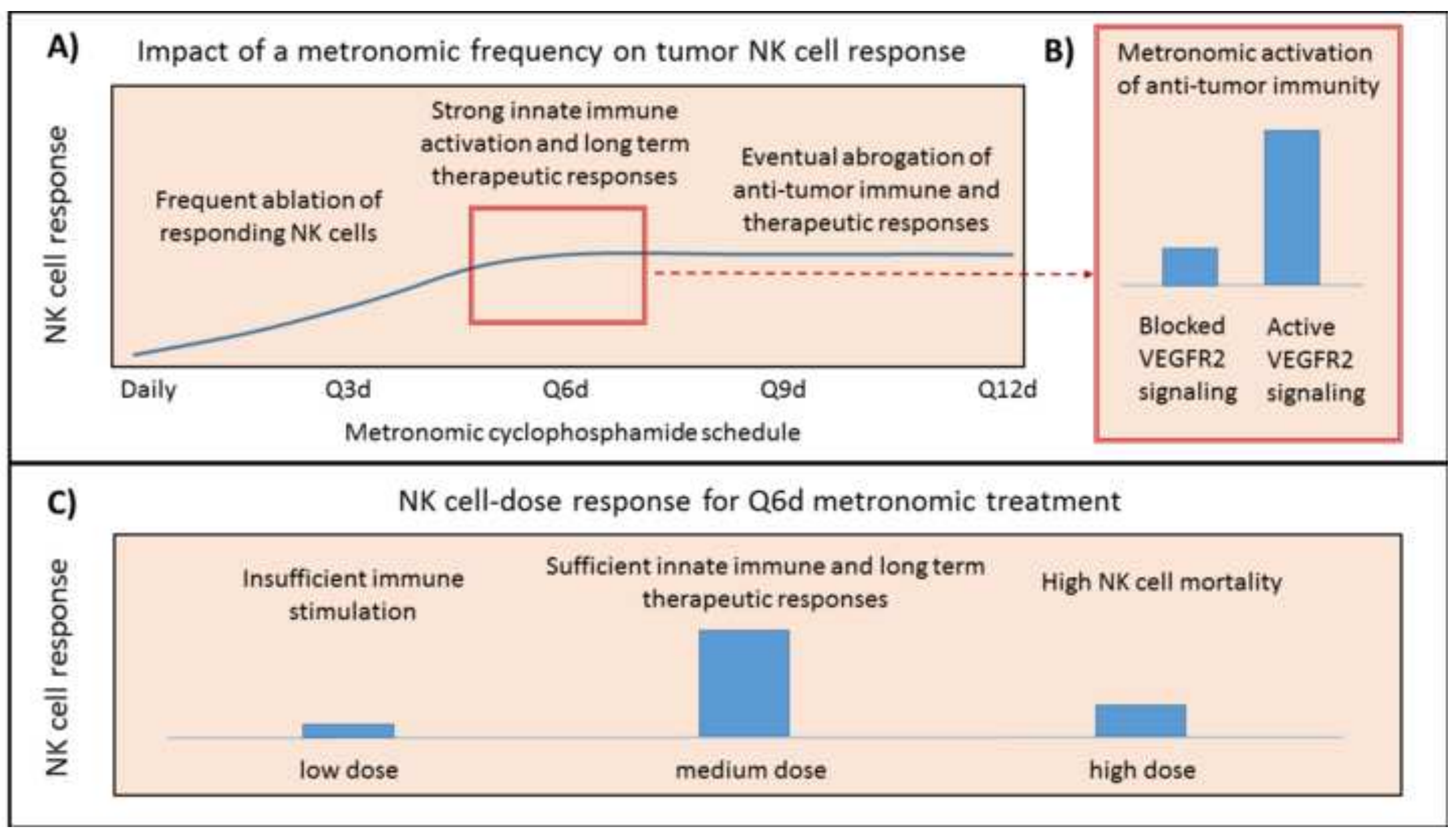

C)

NK cell-dose response for Q6d metronomic treatment 


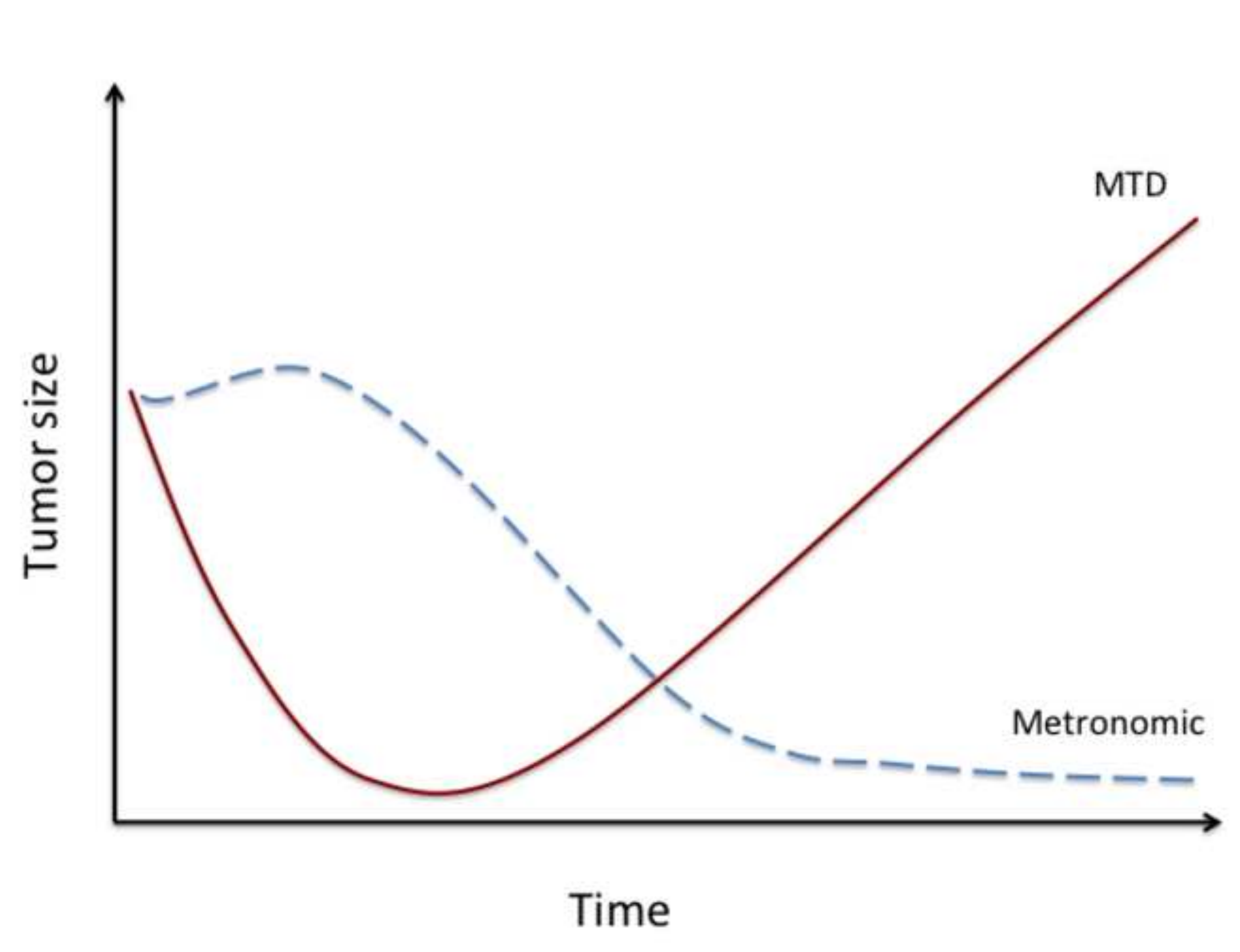

Time

.

.

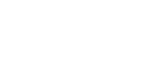

(

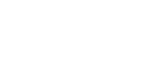

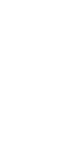

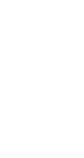

(1)
. 